\title{
Exposures of Phenylacetic Acid and Phenylacetylglutamine Across Different Subpopulations and Correlation with Adverse Events
}

\author{
Xiaofeng Wang ${ }^{1} \cdot$ Jack Tseng $^{2} \cdot{\text { Carmen } \text { Mak }^{2} \cdot \text { Nagaraju Poola }^{1} \cdot \text { Regis A. Vilchez }}^{2}$
}

Accepted: 1 June 2021 / Published online: 14 June 2021

(c) The Author(s) 2021

\begin{abstract}
Background Elevated plasma ammonia is central to the pathogenesis of hepatic encephalopathy. Sodium phenylacetate or glycerol phenylbutyrate is approved for urea cycle disorders, but limited clinical data are available for hepatic encephalopathy. Phenylacetic acid (PAA) plasma exposure has been reported to correlate with neurologic adverse events in patients with cancer but not in patients with urea cycle disorders or hepatic encephalopathy. Ornithine phenylacetate, an intravenous dosage form of the L-ornithine salt of phenylacetate, is under development for hepatic encephalopathy.

Objective This analysis summarized the pharmacokinetics and safety of ornithine phenylacetate to support the dosing strategy and to assist with the monitoring and management of neurologic adverse events in a global clinical development program. Methods Phenylacetic acid and phenylacetylglutamine (PAGN) pharmacokinetic data and adverse events from five clinical studies were included in the analysis. Hepatic and renal dysfunction were assessed by baseline Child-Pugh score and creatinine clearance, respectively. Predicted plasma exposures of PAA at the occurrence of neurologic adverse events were used for exposures and neurologic adverse event analysis.

Results Phenylacetic acid exhibited nonlinear pharmacokinetics. Phenylacetic acid exposure was 35\% higher in Child-Pugh $\mathrm{C}$ than in Child-Pugh B. No significant pharmacokinetic difference was identified between Caucasian and Asian subjects after body weight adjustment. Phenylacetylglutamine renal clearance decreased by five-fold in severe renal impairment compared with subjects with normal renal function. Renal dysfunction significantly elevated PAGN plasma concentrations; however, elevated PAGN due to reduced excretion of PAGN did not change PAA exposure and plasma ammonia levels. No correlation was observed between PAA plasma exposure and neurologic adverse events in patients with stable cirrhosis or acute hepatic encephalopathy.

Conclusions Dose adjustment should be considered for patients with low body weight and severely impaired hepatic function. Phenylacetic acid plasma exposure was not correlated with neurologic adverse events in the ornithine phenylacetate target patient population.
\end{abstract}

\section{Introduction}

Hepatic encephalopathy (HE) is a debilitating neurocognitive disorder that arises in the setting of chronic progressive liver disease [1]. Although the mechanism of $\mathrm{HE}$

Xiaofeng Wang, Carmen Mak, Nagaraju Poola: Affiliation at the time that the research was conducted.

Regis A. Vilchez

regis.vilchez@mnk.com

1 Clinical Pharmacology and Pharmacometrics, Mallinckrodt Pharmaceuticals, Hampton, NJ, USA

2 Clinical Development, Mallinckrodt Pharmaceuticals, Hampton, NJ 08827, USA development is unclear, available evidence points to a central role of elevated ammonia in its pathogenesis [2]. Phenylacetic acid (PAA) is a well-characterized ammonia scavenger [3] and is commonly formulated as a sodiumbased or potassium-based salt to enhance aqueous solubility. Products that contain PAA or phenylbutyrate as a primary active moiety after metabolism, which include sodium phenylbutyrate and glycerol phenylbutyrate, are approved for the acute and/or maintenance treatment of patients with urea cycle disorders [4-6] but not indicated for use in patients with acute HE.

Ornithine phenylacetate (L-OPA) is an intravenous dosage form of the L-ornithine salt of PAA. Once administered, L-OPA dissociates to PAA and ornithine (ORN). Ornithine is an endogenous non-proteogenic amino acid involved in 


\section{Key Points}

This analysis was performed to evaluate whether neurologic adverse events (AEs) are associated with plasma concentrations of phenylacetic acid (PAA) in patients with hepatic encephalopathy who are treated by ornithine phenylacetate. We concluded that PAA exposure did not appear to correlate with neurologic AEs in patients with stable cirrhosis or acute hepatic encephalopathy. This work has significant impact as the current PAAbased drug label stated that PAA is associated with neurologic AEs

The analysis was also conducted to assist phase III dose selection in the global clinical development program. The analysis concluded that body weight and hepatic dysfunction had a significant impact on the exposure of PAA; while ethnicity after body weight adjustment was not a statistically significant factor that affects PAA exposure. However, although ethnicity has no direct impact on PAA exposure, PAA exposure in Asian patients may be higher than in Caucasian patients at the same dose concentration, owing to the lower average body weight of Asian patients

Impairment of kidney function substantially reduced the excretion of phenylacetylglutamine (PAGN, a metabolite of PAA), but this did not affect plasma concentrations of PAA or ammonia level reduction. This confirms that the formation of PAGN is an irreversible process. Furthermore, elevated PAGN exposure due to renal dysfunction did not appear to increase the rate of AE occurrence

protein metabolism and in the urea cycle [7]. It is normally present in human plasma in the range of $4-14 \mu \mathrm{g} / \mathrm{mL}$ and is combined with ammonia during glutamine synthesis in skeletal muscle [8-10]. Free PAA exerts its therapeutic effect by conjugating with glutamine in the liver to form phenylacetylglutamine (PAGN), which is excreted by the kidney [9]. The formation of glutamine from glutamate is reversible, potentially allowing ammonia to be released back to the systemic circulation; however, when PAA covalently binds to glutamine to form PAGN, it is considered to be an irreversible reaction [9]. In addition to its potential role in increasing the glutamate pool, ORN serves as a pH-neutral and electrolyte-neutral counterion for the delivery of PAA [9]. The metabolism of PAA and the route of elimination of PAGN suggest that hepatic impairment is likely to alter exposure to PAA, whereas renal dysfunction is likely to alter exposure to PAGN [11].

Neurologic adverse events (AEs) have been reported to correlate with PAA plasma concentration at approximately $\geq 490 \mu \mathrm{g} / \mathrm{mL}$ in patients with cancer receiving sodium phenylacetate intravenously [12]. However, Mokhtarani et al. [13] concluded that, in contrast to healthy volunteers, elevated PAA exposures did not correlate with neurologic AEs in patients with urea cycle disorders or HE. The authors recommended that additional clinical data be obtained to confirm their conclusion because of limitations to their observations such as the pharmacokinetic blood samples not being collected at the time of the occurrence of AEs.

The overall objective of the current analysis was to summarize the findings of pharmacokinetics (PK) and safety at different doses and across different patient subpopulations. This was to provide data to support the rationale of the dosing strategy for the global phase III L-OPA clinical program in HE, and particularly to assist in the monitoring and management of central nervous system (CNS) AEs during the clinical study. To achieve this objective, factors that could alter exposure to PAA and PAGN in patients were assessed; these factors included body weight, ethnicity, and hepatic/ renal dysfunction. The potential correlation between PAA plasma exposure and neurologic AEs (i.e., CNS AEs) was evaluated. Furthermore, potential risk of PAGN accumulation in plasma due to renal dysfunction was explored.

\section{Methods}

The pharmacokinetic and neurologic AE data are from five clinical studies, described below. An institutional review board (Table S1 of the Electronic Supplementary Material [ESM]) approved each study protocol. All participants gave written informed consent before the initiation of study procedures.

\subsection{Clinical Study Description}

HV201 (Protocol, OCR002-HV201), the first study of L-OPA in healthy volunteers, comprised two parts: single ascending dose (SAD) and multiple ascending dose. The investigated doses in the SAD part were $1,10,20$, and $30 \mathrm{~g}$ with $4 \mathrm{~h}$ of infusion, and 30, 40, and 60 with $24 \mathrm{~h}$ of infusion; in the multiple ascending dose part, doses were 1,3 , 10 , and $20 \mathrm{~g}$ with $24 \mathrm{~h}$ of infusion administered for 5 days.

HE201 (Protocol, OCR002-HE201) was a SAD study in patients with stable cirrhosis (Child-Pugh A, $n=31$; Child-Pugh B, $n=12$ ) to evaluate the tolerability and PK of L-OPA. Tested doses were $1,3,10,20$, and $40 \mathrm{~g}$ with $4 \mathrm{~h}$ of infusion, and 10, 20, and $40 \mathrm{~g}$ with $24 \mathrm{~h}$ of infusion.

HE209 (Protocol, OCR002-HE209) was a phase IIb, placebo-controlled, randomized, double-blind study (ClinicalTrials.gov NCT01966419) to evaluate the safety, PK, and efficacy of L-OPA in hospitalized patients with liver cirrhosis and an acute episode of HE (Child-Pugh A, $n=3$; 
Child-Pugh B, $n=66$; Child-Pugh C, $n=162$ ) [14]. Either L-OPA or placebo was administered to patients as a continuous intravenous infusion for up to 5 days together with standard of care. Dosage for each patient was based on baseline calculations of hepatic synthetic and portal elements (4-6 points, $20 \mathrm{~g} / 24 \mathrm{~h}$; 7-9 points, $15 \mathrm{~g} / 24 \mathrm{~h}$; and $10-12$ points, $10 \mathrm{~g} / 24 \mathrm{~h}$ ).

MNK61051112 (Protocol, MNK61051112) was an openlabel study to assess the PK and safety of L-OPA in healthy Chinese Han and Japanese volunteers receiving a dose of $20 \mathrm{~g}$ with $24 \mathrm{~h}$ of infusion. The study was conducted to support the L-OPA development program for Asia.

MNK61051111 (Protocol, MNK61051111) was an open-label study to assess the PK and safety following the administration of L-OPA in adults with severe renal impairment. This study was conducted to support the inclusion of patients with severely impaired renal function in the phase III clinical trial; prior to this study, pharmacokinetic and AE information was only available from the two clinical studies that included patients with mild/moderate renal dysfunction. The infused dose was $7 \mathrm{~g} / 24 \mathrm{~h}$ for patients with severe renal impairment and $15 \mathrm{~g} / 24 \mathrm{~h}$ for the control group including healthy subjects (normal renal function). Patients in the renal impairment and normal renal function groups were matched by sex, body weight, and age.

\subsection{Pharmacokinetic Analysis}

Blood samples for full pharmacokinetic profiles of ORN, PAA, and PAGN were collected from all studies except study HE209. In study HE209, sparse pharmacokinetic samples were collected before the first dose, at the end of the 24-hour infusion of each dose (total up to five infusions), and at 0.5 , 1 , and $3 \mathrm{~h}$ after the end of the last infusion. Plasma concentrations of ORN, PAA, and PAGN and urine concentrations of PAGN were determined using a validated liquid chromatography-tandem mass spectrometry method [11].

Maximum concentration $\left(C_{\max }\right)$, area under the concentration-time curve (AUC), clearance (CL), and elimination half-life of PAA were estimated using plasma ORN, PAA, and PAGN concentration data from studies with full pharmacokinetic profiles. For study HE209, steady-state concentrations of PAA following 5 days of continuous infusion were summarized by dose concentration and Child-Pugh category.

The impact of hepatic dysfunction on PAA PK was investigated using the pharmacokinetic data from patients in studies HE201 and HE209. The impact of renal dysfunction on PAA and PAGN plasma exposure was explored using the pharmacokinetic data from studies HE201, HE209, and MNK61051111. The effect of ethnicity on the PK of PAA was evaluated using pharmacokinetic data from Caucasian,
Chinese, and Japanese healthy volunteers in studies HV201 and MNK61051112.

In addition, PAA, PAGN, and ammonia plasma concentrations were compared between patients in the HE209 study with creatinine CL (CLcr) $\leq 35$ and $>35 \mathrm{~mL} / \mathrm{min}$, a cut-off chosen to ensure a sufficient sample size for the group with worsened renal function and to allow for a balanced distribution of patients with hepatic dysfunction. For example, of the nine patients in the study with CLcr $\leq 35 \mathrm{~mL} / \mathrm{min}$, six patients were Child-Pugh C, two were Child-Pugh B, and one was Child-Pugh A. The distribution of Child-Pugh C patients in this subgroup $(67 \%)$ was similar to the subgroup with baseline CLcr $>35 \mathrm{~mL} / \mathrm{min}(66 \%)$.

During the analysis to assess the impact of ethnicity on the PK of PAA, it was discovered that confounding factors such as body weight and sex could have an impact on the observed difference in PAA exposure. Therefore, population pharmacokinetic modeling was adopted to evaluate ethnicity as a factor on the PK of PAA using PAA plasma concentrations from healthy Caucasian and Asian healthy volunteers.

\subsection{AE Assessment and PAA Exposure Relationship with CNS AEs and AEs Related to Abuse of the Study Drug}

Adverse events were assessed and coded using the Medical Dictionary for Regulatory Activities preferred term and system organ class. Central nervous system-related events monitored for a possible relationship to L-OPA included somnolence, fatigue, lightheadedness, headache, dysgeusia, hypoacusis, disorientation, impaired memory, and peripheral neuropathy.

The evaluation of a potential correlation between all drug-related or possibly related CNS AEs and PAA exposure was performed with the pharmacokinetic and safety data from all studies. For each such CNS AE, PAA concentrations corresponding to the AEs were imputed using the individual prediction with empirical Bayes parameters from a population pharmacokinetic model for PAA (data on file). For comparison, an analysis using the observed PAA concentration at the nearest $\mathrm{AE}$ occurrence was also conducted. All time points of CNS AE occurrence were calculated relative to the start of study drug infusion. Following the same procedures, a correlation between PAA or PAGN exposure with AEs of drug abuse was also explored.

Noncompartmental analysis was conducted using Phoenix ${ }^{\circledR}$ WinNonlin ${ }^{\circledR}$ Version 6.4 (Certara Inc., Princeton, NJ, USA), and a population pharmacokinetic analysis was conducted using NONMEM Version 7.4 (ICON, Ellicott City, MD, USA). Data processing including figures and a statistical summary was performed using R Version 3.5. 


\section{Results}

Participant demographics and baseline disease characteristics for patients are summarized in Tables 1 and 2.

\subsection{PK of PAA}

Phenylacetic acid demonstrated nonlinear PK with a greater than dose-proportional increase in $\mathrm{C}_{\max }$ and AUC in healthy volunteers from study HV201 and patients with stable cirrhosis in study HE201. Figure 1 shows the relationship between dose and $\mathrm{AUC}_{\text {inf }}$ following single-dose administration, either over 4 or $24 \mathrm{~h}$ of infusion in healthy subjects or in patients with stable cirrhosis. An over-proportional dose-dependent increase in exposure was observed at doses $\geq 20 \mathrm{~g}$, either with 4 or $24 \mathrm{~h}$ of infusion.

In healthy volunteers, the geometric mean elimination half-life for PAA increased from 0.65 to $5.99 \mathrm{~h}$ when the dose was increased from 3 to $30 \mathrm{~g}$ with $4 \mathrm{~h}$ of infusion, and from 1.25 to $4.09 \mathrm{~h}$ when the dose was increased from 30 to $60 \mathrm{~g}$ with $24 \mathrm{~h}$ of infusion. In patients with stable cirrhosis, the geometric mean elimination half-life increased from 0.81 to $6.06 \mathrm{~h}$ with an increase in dose from 3 to $40 \mathrm{~g}$ by a 4-hour infusion, and from 1.64 to $3.35 \mathrm{~h}$ with an increase in dose from 10 to $40 \mathrm{~g}$ by a 24-hour infusion.

\subsection{Effect of Hepatic and Renal Dysfunction on PAA and PAGN PK}

Patients in study HE201 and HE209 had various degrees of hepatic (Child-Pugh A/B/C) and/or renal impairment. Because of missing ethnicity information of some patients in study HE209, estimated glomerular filtration rate could not be derived for all patients. Therefore, baseline CLcr was used to indicate the degree of renal impairment.

Hepatic dysfunction significantly altered plasma PAA concentrations. In study HE209, pharmacokinetic data were available from 38 patients with Child-Pugh $\mathrm{C}$ and nine patients with Child-Pugh B who were administered $15 \mathrm{~g}$ of L-OPA with 24 h of infusion. The median plasma PAA concentration at steady state was approximately $35 \%$ higher in Child-Pugh C patients $(120 \mu \mathrm{g} / \mathrm{mL})$ than that of Child-Pugh B $(89.1 \mu \mathrm{g} / \mathrm{mL})$ (Table 3$)$. The median plasma PAA exposure in patients with Child-Pugh B at $20 \mathrm{~g}$ was $36 \%$ higher (163 vs $120 \mu \mathrm{g} / \mathrm{mL}$ ) than that of patients with Child-Pugh C at the 15-g dose. No difference in safety profiles was observed in the 20-g dose cohort (see safety results). Therefore, the selected phase III dose of $15 \mathrm{~g}$ of L-OPA with $24 \mathrm{~h}$ of infusion was applied to patients with normal liver function to Child-Pugh C for Caucasian patients.

Renal dysfunction did not appear to affect PAA exposure (Fig. 2a) but significantly increased the plasma concentration
Table 1 Summary of demographics in healthy volunteers (HV201)

$\begin{array}{llll}\text { Weight (kg) } & \text { Age (years) } & \text { Sex, } n(\%) & \text { Race, } n(\%) \\ n & n & \text { Male } & \text { Asian } \\ \text { Mean (SD) } & \text { Mean (SD) } & \text { Female } & \text { African } \\ & & & \text { American/ } \\ & & & \text { black } \\ & & & \text { Caucasian/ } \\ & & & \text { white } \\ & & \text { Missing }\end{array}$

\begin{tabular}{lllll}
\hline $\begin{array}{l}\text { L-OPA } \\
\text { SAD }\end{array}$ & 16 & 16 & $7(43.75)$ & 0 \\
$(N=16)$ & $71.5(7.7)$ & $26.4(9.9)$ & $9(56.25)$ & 0 \\
& & & & $15(93.75)$ \\
& & & & $1(6.25)$ \\
L-OPA & 20 & 20 & $4(20.00)$ & 0 \\
MAD & $70.3(10.2)$ & $26.1(10.0)$ & $16(80.00)$ & $1(5.00)$ \\
$(N=20)$ & & & & $18(90.00)$ \\
& & & & $1(5.00)$ \\
Placebo & & & $2(25.00)$ & $1(12.50)$ \\
SAD & 8 & 8 & $6(75.00)$ & 0 \\
$(N=8)$ & $69.8(5.6)$ & $32.0(14.3)$ & & $7(87.50)$ \\
& & & & 0 \\
& & & $2(50.00)$ & 0 \\
Placebo & 4 & 4 & $2(50.00)$ & 0 \\
MAD & $72.2(8.4)$ & $33.0(17.1)$ & & $4(100.00)$ \\
$(N=4)$ & & & & 0
\end{tabular}

L-OPA ornithine phenylacetate, MAD multiple ascending dose, $S A D$ single ascending dose, $S D$ standard deviation

of PAGN. Plasma concentrations of PAA and PAGN for the two subgroups (CLcr $\leq 35$ or $>35 \mathrm{~mL} / \mathrm{min}$, balanced in hepatic dysfunction) are plotted in Fig. 2a. Locally estimated scatterplot smoothing fitting suggested that renal dysfunction does not alter the PAA profiles but significantly elevated the PAGN plasma concentration in those patients with CLcr $\leq 35 \mathrm{~mL} / \mathrm{min}$. In addition, renal dysfunction did not alter ammonia level (Fig. 2b).

To further evaluate renal dysfunction on L-OPA administration, a dedicated renal impairment study (MNK61051111) was conducted to evaluate the impact of severe renal impairment on the PK of PAA and PAGN. Age and body weight distribution of the two groups were matched. Because PAA shows nonlinear PK at doses of approximately $\geq 20 \mathrm{~g}$ in healthy subjects, if renal dysfunction were not to have an impact on the exposure of PAA and PAGN, dose-normalized exposure of PAA and PAGN for the two groups, normal renal function at $15 \mathrm{~g}$ vs severe renal dysfunction at $7.5 \mathrm{~g}$, would be comparable. Pharmacokinetic parameters derived from the noncompartmental analysis showed that the mean dose-normalized PAA $\mathrm{C}_{\max }$ and AUC from time 0 to time of last quantifiable concentration $\mathrm{t}\left(\mathrm{AUC}_{\mathrm{t}}\right)$ were $2.61 \mu \mathrm{g} /$ $\mathrm{mL} / \mathrm{g}$ and $54.9 \mu \mathrm{g} \bullet \mathrm{h} / \mathrm{mL} / \mathrm{g}$ with normal renal function, and $1.58 \mu \mathrm{g} / \mathrm{mL} / \mathrm{g}$ and $45.4 \mu \mathrm{g} \bullet \mathrm{h} / \mathrm{mL} / \mathrm{g}$ with severely impaired 
Table 2 Summary of demographics and baseline information in patients with stable liver cirrhosis (HE201), in those with liver cirrhosis admitted to the hospital because of an acute episode of

\begin{tabular}{|c|c|c|c|c|c|c|c|}
\hline Study & Variable & $\begin{array}{l}\text { Weight }(\mathrm{kg}) \\
n \\
\text { Mean (SD) }\end{array}$ & $\begin{array}{l}\text { Age (years) } \\
n \\
\text { Mean (SD) }\end{array}$ & $\begin{array}{l}\text { Sex, } n(\%) \\
\text { Male } \\
\text { Female }\end{array}$ & Race & $\begin{array}{l}\text { Child-Pugh } \\
n\end{array}$ & $\begin{array}{l}\text { Renal function } \\
n\end{array}$ \\
\hline $\begin{array}{l}\text { Patients with cirrhosis } \\
\text { (HE201) }\end{array}$ & & $\begin{array}{l}43 \\
81.1(15.3)\end{array}$ & $\begin{array}{l}43 \\
53.4(7.4)\end{array}$ & $\begin{array}{l}16(37.2) \\
27(62.8)\end{array}$ & $\begin{array}{l}\text { American Indian or } \\
\text { Alaskan Native: 1; } \\
\text { Asian: 3; Caucasian/ } \\
\text { white: } 39\end{array}$ & $\begin{array}{l}\text { A: } 31 \\
\text { B: } 12 \\
\text { C: } 0\end{array}$ & $\begin{array}{l}\text { Mild: } 9 \\
\text { Normal: } 34\end{array}$ \\
\hline \multirow[t]{2}{*}{$\begin{array}{l}\text { Hospitalized patients } \\
\text { with HE (HE209) }\end{array}$} & Placebo & $\begin{array}{l}112 \\
84.6(21.6)\end{array}$ & $\begin{array}{l}115 \\
60(9.5)\end{array}$ & $\begin{array}{l}78(68) \\
37(32.2)\end{array}$ & $\begin{array}{l}\text { Asian: 1; Caucasian/ } \\
\text { white: 54; African } \\
\text { American/black: } 8 \text {; } \\
\text { Native Hawaiian or } \\
\text { Pacific Islander: 1; } \\
\text { Other: } 1 \\
\text { Missing: } 50\end{array}$ & $\begin{array}{l}\text { A: } 1 \\
\text { B: } 28 \\
\text { C: } 86\end{array}$ & $\begin{array}{l}\text { Severe: } 1 \text { Moderate: } 31 \\
\text { Mild: } 36 \\
\text { Normal: } 43 \\
\text { Missing: } 4\end{array}$ \\
\hline & L-OPA & $\begin{array}{l}113 \\
82.1(22.4)\end{array}$ & $\begin{array}{l}116 \\
59.0(9.8)\end{array}$ & $\begin{array}{l}72(62) \\
44(38)\end{array}$ & $\begin{array}{l}\text { American Indian or } \\
\text { Alaskan Native: 1; } \\
\text { Asian: 1; Caucasian/ } \\
\text { white: 58; African } \\
\text { American/black: 5; } \\
\text { Other: } 1 \\
\text { Missing: } 50\end{array}$ & $\begin{array}{l}\text { A: } 2 \\
\text { B: } 38 \\
\text { C: } 76\end{array}$ & $\begin{array}{l}\text { Severe: } 3 \\
\text { Moderate: } 32 \text { Mild: } 35 \\
\text { Normal: } 44 \\
\text { Missing: } 2\end{array}$ \\
\hline \multirow[t]{2}{*}{$\begin{array}{l}\text { Healthy volunteer } \\
\text { (MNK61051112) }\end{array}$} & Chinese Han & $\begin{array}{l}7 \\
75.3(9.1)\end{array}$ & $\begin{array}{l}7 \\
40.4(8.5)\end{array}$ & $7(100.0)$ & Asian: 7 & & Normal: 8 \\
\hline & Japanese & $\begin{array}{l}8 \\
65.4(13.4)\end{array}$ & $\begin{array}{l}8 \\
44.8(11.4)\end{array}$ & $\begin{array}{l}2(25.0) \\
6(75.0)\end{array}$ & Asian: 8 & & $\begin{array}{l}\text { Mild: } 1 \\
\text { Normal: } 7\end{array}$ \\
\hline \multirow[t]{2}{*}{$\begin{array}{l}\text { Renal impairment } \\
\text { (MNK61051111) }\end{array}$} & Normal & $\begin{array}{l}7 \\
87.7(10.3)\end{array}$ & $\begin{array}{l}7 \\
62.0(8.4)\end{array}$ & $\begin{array}{l}5(71.4) \\
2(28.6)\end{array}$ & Caucasian/white: 7 & & Normal: 7 \\
\hline & SRI & $\begin{array}{l}7 \\
84.8(9.2)\end{array}$ & $\begin{array}{l}7 \\
59.1(9.6)\end{array}$ & $\begin{array}{l}5(71.4) \\
2(28.6)\end{array}$ & $\begin{array}{l}\text { Caucasian/white: 5; } \\
\text { African American/ } \\
\text { black: } 2\end{array}$ & & Severe: 7 \\
\hline
\end{tabular}

$H E$ hepatic encephalopathy, $L-O P A$ ornithine phenylacetate, $S D$ standard deviation, $S R I$ severe renal impairment

renal function, respectively. Mean dose-normalized PAGN $\mathrm{C}_{\max }$ and $\mathrm{AUC}_{\mathrm{t}}$ was substantially lower with normal renal function $(3.35 \mu \mathrm{g} / \mathrm{mL} / \mathrm{g}$ and $69.9 \mu \mathrm{g} \bullet \mathrm{h} / \mathrm{mL} / \mathrm{g})$ compared with severely impaired renal function $(16.1 \mu \mathrm{g} / \mathrm{mL} / \mathrm{g}$ and $383 \mu \mathrm{g} \bullet \mathrm{h} / \mathrm{mL} / \mathrm{g}$ ), respectively. Renal CL for PAGN was 24.5 $\mathrm{L} / \mathrm{h}$ with normal renal function and $4.66 \mathrm{~L} / \mathrm{h}$ with severe renal impairment, a five-fold decrease.

\subsection{Effect of Ethnic Factors on PAA PK}

Direct comparison of PAA pharmacokinetic exposure $\left(\mathrm{C}_{\max }\right.$ and AUC derived from noncompartmental analysis) across Caucasian healthy volunteers (study HV201) compared with Chinese Han and Japanese healthy volunteers (Table 4; study MNK61051112) to assess the effect of ethnic factors on PAA exposure was confounded by different body weight and unbalanced sex distribution. Therefore, population pharmacokinetic modeling using pharmacokinetic data from healthy Caucasian and Asian (Chinese Han and Japanese) volunteers was conducted. A one-compartment model with
HE (HE209), in healthy Chinese Han and Japanese volunteers (MNK61051112), and adults with SRI (MNK61051111) 

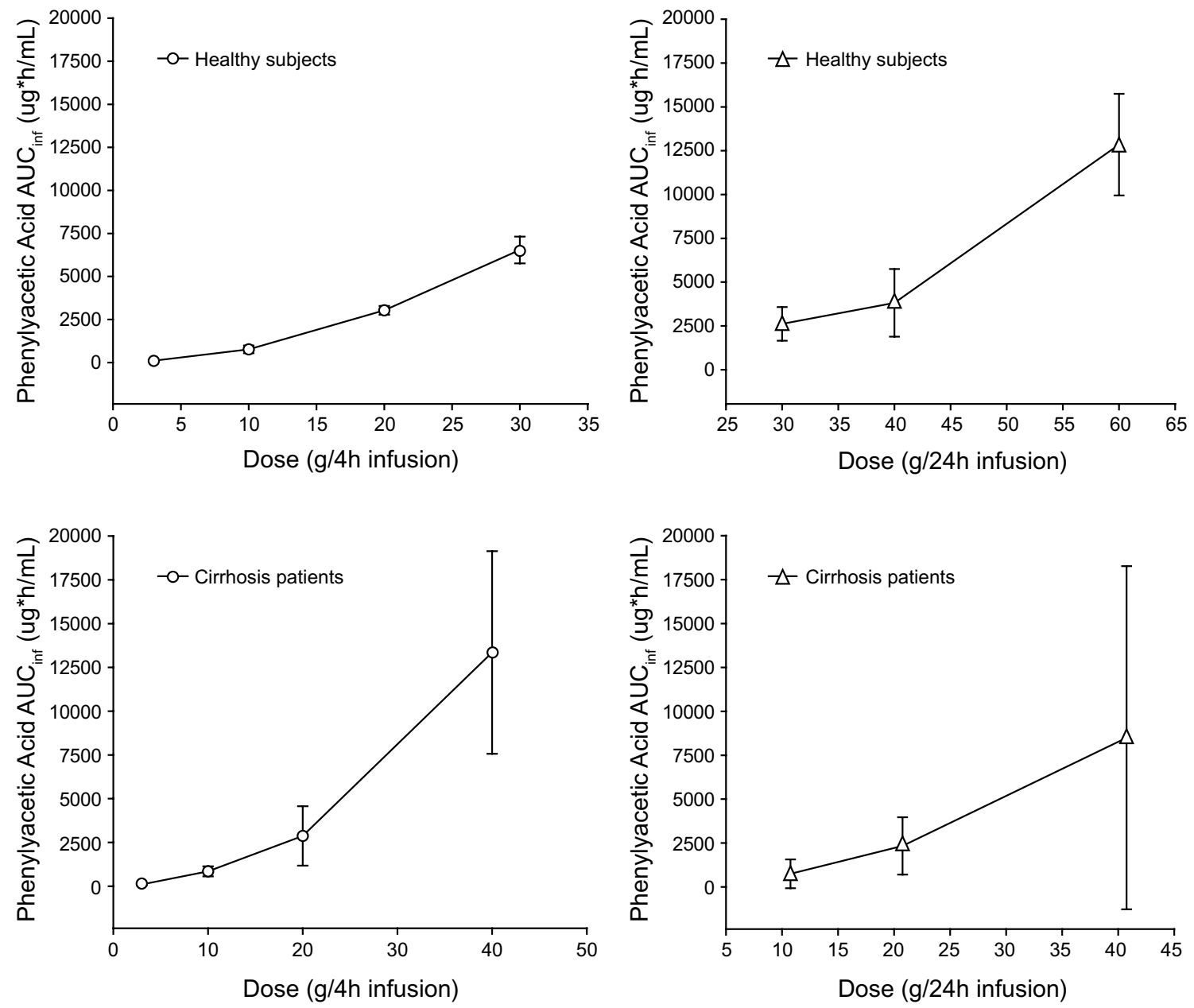

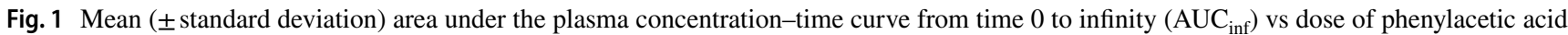
in healthy volunteers (HV201) or in patients with stable cirrhosis in study HE201

Table 3 PAA plasma concentration at steady state in patients with liver cirrhosis admitted to the hospital because of an acute episode of hepatic encephalopathy (HE209)

\begin{tabular}{|c|c|c|c|c|}
\hline \multirow[t]{2}{*}{ Dose } & \multirow[t]{2}{*}{ Statistics } & \multicolumn{3}{|c|}{ Steady-state PAA concentration $(\mu \mathrm{g} / \mathrm{mL})$} \\
\hline & & Child-Pugh A & Child-Pugh B & Child-Pugh C \\
\hline \multirow[t]{4}{*}{$10 \mathrm{~g} / 24 \mathrm{~h}$} & $N$ & & 1 & 23 \\
\hline & Mean & & 43.9 & 104 \\
\hline & $\% \mathrm{CV}$ & & & 51 \\
\hline & Median & & & 118 \\
\hline \multirow[t]{4}{*}{$15 \mathrm{~g} / 24 \mathrm{~h}$} & $N$ & & 9 & 38 \\
\hline & Mean & & 93.7 & 165 \\
\hline & $\% \mathrm{CV}$ & & 54 & 69 \\
\hline & Median & & 89.1 & 120 \\
\hline \multirow[t]{4}{*}{$20 \mathrm{~g} / 24 \mathrm{~h}$} & $N$ & 2 & 19 & \\
\hline & Mean & 153 & 218 & \\
\hline & $\% \mathrm{CV}$ & & 77 & \\
\hline & Median & 153 & 163 & \\
\hline
\end{tabular}

$\% C V$ percent coefficient of variation, $P A A$ phenylacetic acid than other ethnic factors such as genetic polymorphisms of cytochrome P450 enzymes.

\subsection{Safety/Tolerability}

In healthy Caucasian volunteers in the SAD part of study HV201, L-OPA-related treatment-emergent AEs (TEAEs) included headache $(n=19)$, dizziness postural $(n=10)$, nausea $(n=11)$, somnolence $(n=7)$, tinnitus $(n=7)$, vomiting $(n=6)$, and dizziness $(n=4)$. In the multiple ascending dose part of study HV201, L-OPA-related TEAEs included headache $(n=5)$, somnolence $(n=4)$, and tinnitus $(n=4)$; all were reported in the 20-g/4-hour regimen. There were no clinically significant abnormal laboratory parameters, and there were no deaths or serious AEs during the study. Doses $\leq 10 \mathrm{~g} / 4 \mathrm{~h}$ were well tolerated. Dosing with $24 \mathrm{~h}$ of infusion appeared to be a preferred regimen because of tolerability. 
Fig. 2 Effect of renal dysfunction in patients hospitalized with liver cirrhosis and an episode of hepatic encephalopathy in study HE209. a Effect of renal dysfunction on phenylacetic acid (PAA) and phenylacetylglutamine (PAGN) exposure in study HE209. b Effect of renal dysfunction on ammonia level in study HE209. CLcr creatinine clearance, $P A G N p$ plasma concentration of PAGN
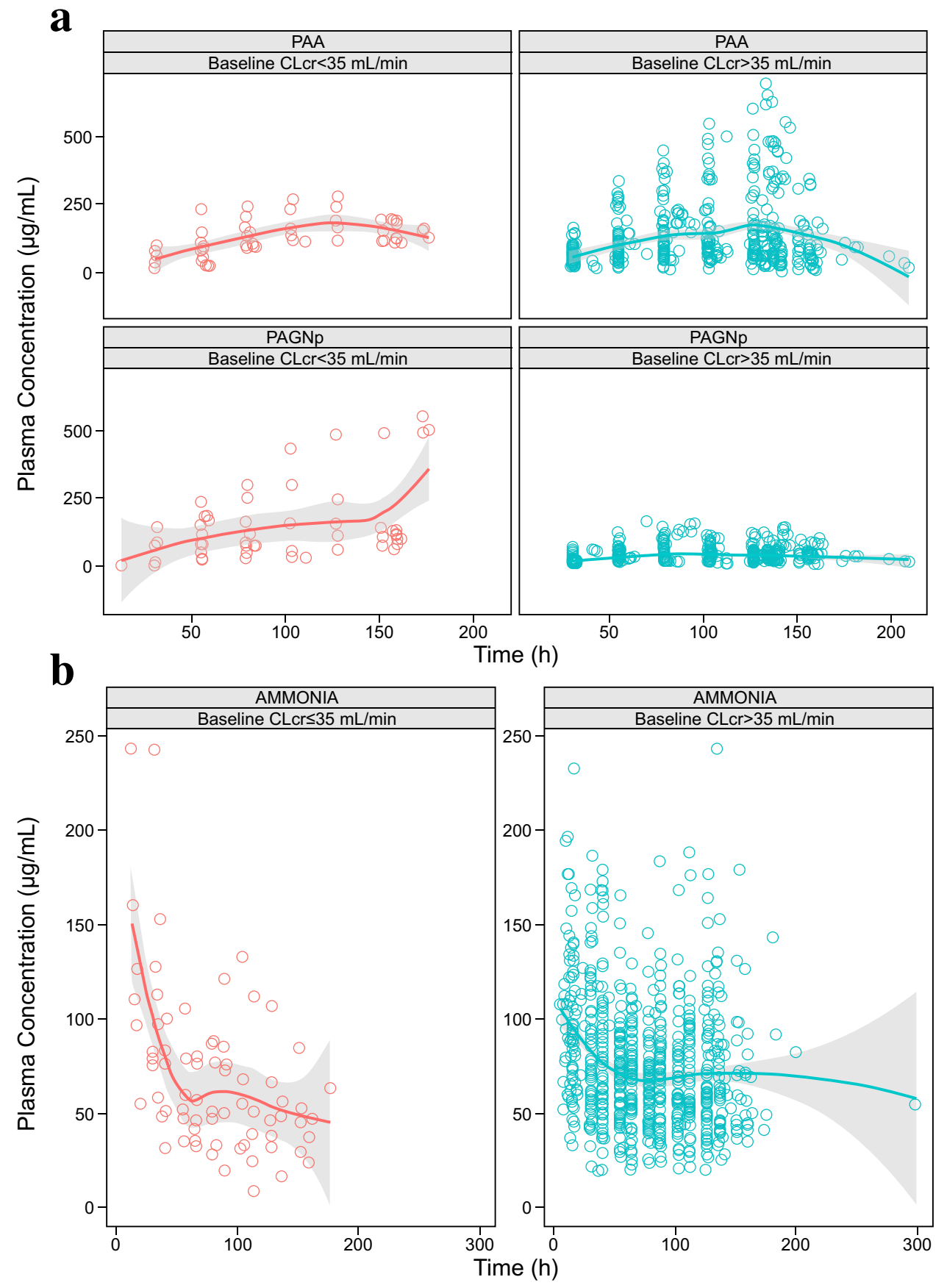

In the single-dose 20-g/24-h study of healthy Chinese Han and Japanese volunteers (MNK61051112), no clinically significant findings in laboratory parameters, vital signs, physical examinations, or electrocardiograms were observed. The only TEAE deemed possibly related to the study drug was mild nausea in a Japanese volunteer, which was consistent with AEs of nausea reported in healthy volunteers in other L-OPA studies. In patients with stable cirrhosis in study HE201, L-OPA was well tolerated up to $20 \mathrm{~g} / 4 \mathrm{~h}$, or $40 \mathrm{~g}$ with a 24-hour infusion. The L-OPA-related TEAEs in the 40-g/24-hour cohort included headache, somnolence, and restlessness.

Safety and efficacy in hospitalized patients with cirrhosis and an acute episode of HE (study HE209) were reported by Rahimi et al. [14]. Incidence-specific AEs between the L-OPA and placebo groups were not statistically significant. Serious AEs occurred in 25\% of the L-OPA group and $29 \%$ of the placebo group. A total of 26 patients (15 placebo, 11 L-OPA) died during the study. The death rates for L-OPA and placebo were $24 \%$ and $26 \%$ for $10 \mathrm{~g} / 24 \mathrm{~h}, 5 \%$ and $12 \%$ 
Table 4 Descriptive summary of pharmacokinetic parameters in healthy Chinese Han and Japanese volunteers (MNK61051112)

\begin{tabular}{|c|c|c|c|c|c|c|}
\hline \multirow[t]{2}{*}{ Group } & \multirow{2}{*}{$\begin{array}{l}\text { Sex, } n \\
\text { Male } \\
\text { Female }\end{array}$} & \multirow{2}{*}{$\begin{array}{l}\text { Weight (kg) } \\
\text { Median }\end{array}$} & \multicolumn{2}{|l|}{ PAA } & \multicolumn{2}{|l|}{ PAGN } \\
\hline & & & $\begin{array}{l}\mathrm{AUC}_{\mathrm{t}}(\mathrm{h} \bullet \mu \mathrm{g} / \mathrm{mL}) \\
\text { Mean }(\mathrm{SD})\end{array}$ & $\begin{array}{l}\mathrm{C}_{\max }(\mu \mathrm{g} / \mathrm{mL}) \\
\text { Mean }(\mathrm{SD})\end{array}$ & $\begin{array}{l}\mathrm{AUC}_{\mathrm{t}}(\mathrm{h} \bullet \mu \mathrm{g} / \mathrm{mL}) \\
\text { Mean }(\mathrm{SD})\end{array}$ & $\begin{array}{l}\mathrm{C}_{\max }(\mu \mathrm{g} / \mathrm{mL}) \\
\text { Mean }(\mathrm{SD})\end{array}$ \\
\hline Chinese Han ${ }^{\mathrm{a}}$ & 7 (all male) & 73.4 & $895(331)$ & $45.4(14.9)$ & 828 (177) & $35.3(9.49)$ \\
\hline Japanese & $\begin{array}{l}2 \text { male } \\
6 \text { female }\end{array}$ & 59.8 & $1590(692)$ & $94.7(35.8)$ & 941 (169) & $41.9(7.31)$ \\
\hline
\end{tabular}

$A U C_{t}$ area under the plasma concentration-time curve from time 0 to time at last quantifiable concentration, $C_{\max }$ maximum plasma concentration, $P A A$ phenylacetic acid, $P A G N$ phenylacetylglutamine, $S D$ standard deviation, $t$ time

${ }^{a}$ One Chinese Han participant was excluded from the noncompartmental pharmacokinetic analysis because of an incomplete pharmacokinetic profile

for $15 \mathrm{~g} / 24 \mathrm{~h}$, and $4 \%$ and $0 \%$ for $20 \mathrm{~g} / 24 \mathrm{~h}$, respectively. In study MNK61051111, a single 7-g or 15-g dose of L-OPA was well tolerated by all participants with severe renal impairment or matched normal renal function, respectively. No L-OPA-related TEAEs were reported.

\subsection{Relationship Between PAA Exposure and CNS AEs}

No CNS AEs were reported from study MNK61051112 in healthy Chinese Han and Japanese volunteers and study MNK61051111 in healthy volunteers and patients with severe renal impairment.

In the healthy volunteer study, HV201, 77 CNS AEs occurred and were considered by the investigators to be study drug related or possibly/remotely related. Twelve CNS AEs were severe, 20 were moderate, and 45 were mild. The reported CNS AEs (headache, dizziness, and somnolence) were included in the exposure of PAA and CNS AE analysis. The severity of the CNS AEs and their corresponding PAA plasma concentration are presented in Fig. 3a. Central nervous system AEs occurred at a wide range of PAA plasma concentrations; at concentrations $>490 \mu \mathrm{g} / \mathrm{mL}$, there were nine CNS AEs (five mild, three moderate, and one severe). No correlation between PAA plasma concentrations and the severity of CNS AEs was observed.

In study HE201, of the 43 patients with stable cirrhosis, 35 received L-OPA, and eight received placebo. Twelve patients receiving L-OPA experienced 24 CNS AEs, including headache, somnolence, dizziness, dysgeusia, and disturbance in attention. Nineteen CNS AEs were of mild severity, and none were severe. The AE severity against the PAA concentration at the occurrence of the AE is plotted in Fig. 3b. One moderate CNS AE was observed at a PAA concentration $>800 \mu \mathrm{g} / \mathrm{mL}$.

Eleven of 226 patients $(4.87 \%)$ with cirrhosis and HE in study HE209 experienced a CNS-related AE. Of these 11 patients, eight received placebo and three received L-OPA. Only two mild CNS AEs (headache) were identified as drug related, both in patients with Child-Pugh $\mathrm{C}$, at PAA concentrations of 90.8 and $208 \mu \mathrm{g} / \mathrm{mL}$. The median concentrations of PAA at steady state for all Child-Pugh C patients treated with L-OPA were $118 \mu \mathrm{g} / \mathrm{mL}$ (range 11.4-223 $\mu \mathrm{g}$ / $\mathrm{mL}$ ) and $120 \mu \mathrm{g} / \mathrm{mL}$ (range $7.15-454 \mu \mathrm{g} / \mathrm{mL}$ ) for patients in the $10-\mathrm{g} / 24-\mathrm{h}(n=23)$ and $15-\mathrm{g} / 24-\mathrm{h}(n=38)$ dose groups, respectively. Thus, the PAA concentrations observed around the time of the CNS AEs were generally around the median. A correlation between PAA exposure and CNS AEs using the observed PAA concentration closest to AE occurrence also indicated that a high PAA concentration was not linked to the severity of drug-related CNS AEs (data not presented).

The mean [standard deviation] PAA concentration at the occurrence of mild CNS AEs appeared to be lower (113 [147] $\mu \mathrm{g} / \mathrm{mL})$ in patients with cirrhosis than that of healthy subjects (259 [144] $\mu \mathrm{g} / \mathrm{mL})$. However, the ranges of PAA concentrations when CNS AEs were reported were similar. No comparison in PAA concentrations for the occurrence of either moderate or severe CNS AEs between healthy subjects and patients with cirrhosis was conducted, owing to limited CNS AE events in patients.

\subsection{Relationship Between PAGN Exposure and AEs}

In study HE209, nine patients with CLcr $<35 \mathrm{~mL} / \mathrm{min}$ were exposed to a much higher PAGN concentrations and continued accumulating PAGN during the 5 days of infusion (Fig. 2a). Three of the nine patients experienced possible drug-related AEs (blood bicarbonate decrease, $n=1$; headache, $n=1$; hypotension, $n=1$ ). None of the three AEs was serious. The AE data suggest that a high PAGN plasma concentration due to worsened renal function did not affect safety. Analysis of PAA or PAGN exposure and AEs of drug abuse conducted using data from study HV201 and study HE201 identified no correlations (Table 5). 
Fig. 3 Phenylacetic acid concentration and severity of central nervous system adverse events. a Phenylacetic acid concentration and severity of central nervous system adverse events in healthy volunteers in study HV201. b Phenylacetic acid concentration and severity of central nervous system adverse events in patients with chronic liver disease (cirrhosis) in study HE201 $\mathbf{a}$

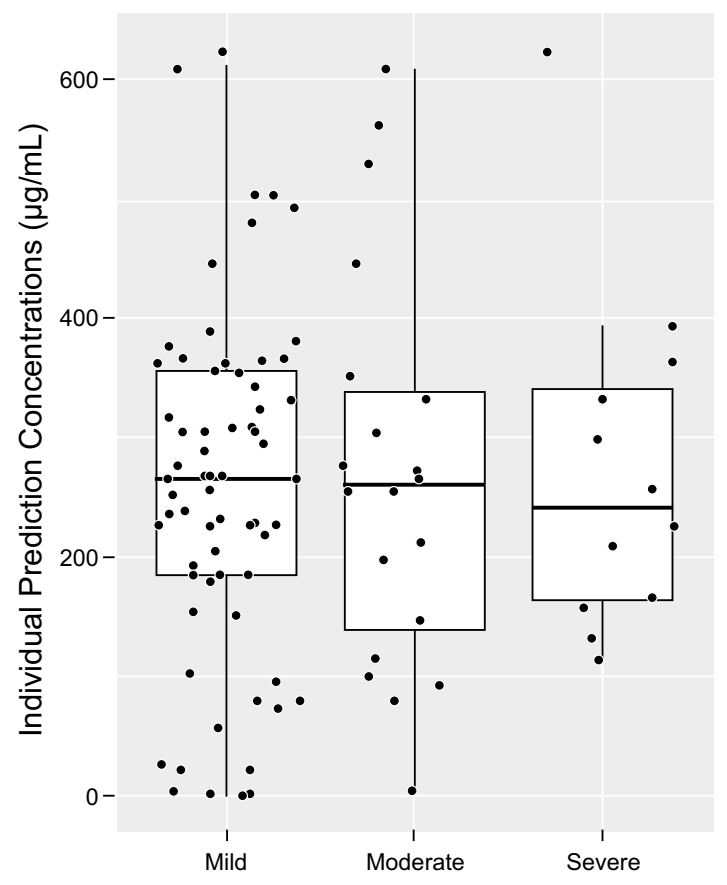

b

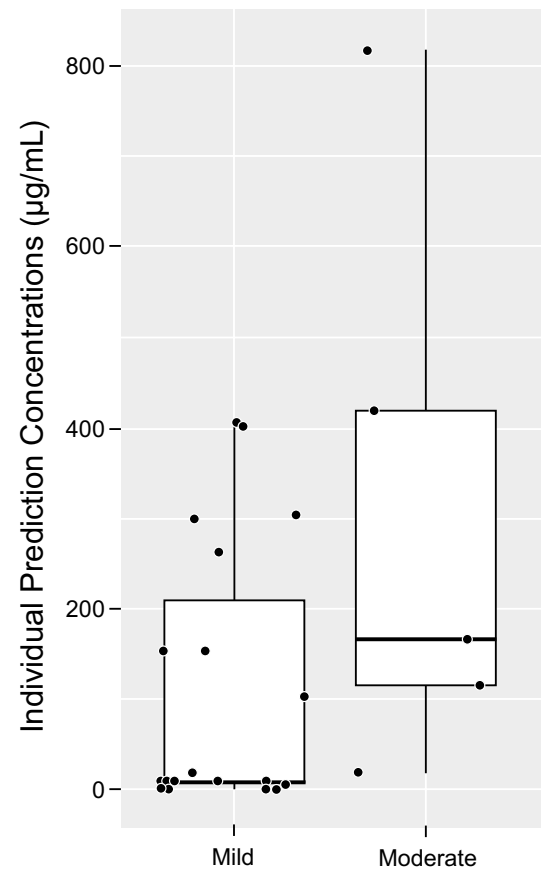

\section{Discussion and Conclusions}

Metabolism of L-OPA does not involve cytochrome P450 enzymes. Ornithine is an amino acid that is a well-known intermediate in various metabolic processes, particularly the urea cycle. It is used as a substrate to produce L-arginine and is recycled back to ORN and urea by arginase. In vitro metabolism and transporter studies using microsomal incubations or cell cultures were conducted for PAA and PAGN. Those studies included cytochrome P450 inhibition and induction or testing of PAA and PAGN as a substrate of various transporters. Results demonstrated that phenylacetate is not a substrate for P-glycoprotein, BCRP,
OATP1B1, OATP1B3, OAT1, or OAT3. Phenylacetylglutamine is not a substrate of OCT2 or multidrug and toxin extrusion (MATE2-K) [data on file]. At a clinically relevant dose of $15 \mathrm{~g}$ with $24 \mathrm{~h}$ of infusion of L-OPA, maximum concentrations of PAA and PAGN are unlikely to interact with P450 substrates. Therefore, genetic polymorphism of the cytochrome $\mathrm{P} 450$ enzymes or transporters will not alter the PK of PAA, nor will it affect cytochrome P450-mediated or transporter-mediated drug-drug interactions. This information likely explains why, once the body weight is corrected, ethnicity has no impact on PAA exposure. As such, dose calculation for sodium phenylbutyrate per the label in Japan [15] is the same as the label in the USA [4].
Table 5 Individual predicted PAA and PAGN concentrations in relation to the severity of AEs of drug abuse in healthy volunteers (HV201) and in patients with stable liver cirrhosis (HE201) ${ }^{\mathrm{a}}$

\begin{tabular}{|c|c|c|c|c|c|c|}
\hline & \multirow[t]{2}{*}{ AE severity } & \multirow[t]{2}{*}{$n$} & \multicolumn{2}{|l|}{ PAA } & \multicolumn{2}{|l|}{ PAGN } \\
\hline & & & Mean (SD) & Median (min, max) & Mean (SD) & Median (min, $\max )$ \\
\hline \multirow{3}{*}{$\begin{array}{l}\text { Healthy } \\
\text { volunteers } \\
\text { (HV201) }\end{array}$} & Mild & 38 & $276(127)$ & $272(0.00,609)$ & $55.5(25.9)$ & $54.2(0.00,117)$ \\
\hline & Moderate & 7 & $273(156)$ & $255(92.3,562)$ & $46.6(31.8)$ & $36.5(11.2,105)$ \\
\hline & Severe & 3 & $381(233)$ & $363(158,622)$ & $67.0(3.14)$ & $66.9(63.8,70.1)$ \\
\hline \multirow{3}{*}{$\begin{array}{l}\text { Patients } \\
\text { with stable } \\
\text { cirrhosis } \\
\text { (HE201) }\end{array}$} & Mild & 16 & $162(225)$ & $63.7(0.00,820)$ & $27.8(25.7)$ & $22.5(0.00,67.8)$ \\
\hline & Moderate & 3 & $366(391)$ & $167(115,817)$ & $24.7(23.7)$ & $15.6(6.93,51.6)$ \\
\hline & Severe & 0 & & & & \\
\hline
\end{tabular}

$A E$ adverse event, $\max$ maximum, $\min$ minimum, $n$ number of events, $P A A$ phenylacetic acid, $P A G N$ phenylacetylglutamine, $S D$ standard deviation

${ }^{a}$ In study HE209, there was 1 mild AE of drug abuse that occurred at PAA and PAGN concentrations of $187 \mu \mathrm{g} / \mathrm{mL}$ and $512 \mu \mathrm{g} / \mathrm{mL}$, respectively 
The most significant covariates that alter PAA plasma exposure are body size (weight or body surface area) and hepatic function. The contribution of body size to PAA plasma exposure is known [16]. Therefore, the dose for PAA-based drugs such as sodium phenylbutyrate is calculated using body surface area. In the L-OPA target patient population, severity of liver impairment often corresponds with severity of HE. Observed plasma PAA concentrations from study HE209 confirmed that PAA exposure increased with the severity of hepatic impairment.

Ethnicity had no statistically significant effect on the PK of PAA. Once corrected for body weight and sex, no difference in PK was observed between Caucasian and Asian healthy participants. Notably, sex contributed to the volume of distribution of PAA but not its CL. This indicated that the steady-state plasma PAA concentration would be comparable between male and female patients with the same body weight and liver function, as steady-state plasma PAA concentration is determined by the CL. In study HE209, safety data from patients with a body weight range of $45-153 \mathrm{~kg}$ demonstrated no dose-dependent or exposure-dependent AEs. Phenylacetic acid exposure in patients with severe hepatic impairment administered at $15 \mathrm{~g} / 24 \mathrm{~h}$ was below those observed from patients with moderate hepatic impairment at $20 \mathrm{~g} / 24 \mathrm{~h}$ and was confirmed to be safe. Although ethnicity has no statistically significant impact on the PK of PAA, PAA exposure in Asian patients is expected to be higher because of their low body weight compared with PAA exposure at the same dose as for Caucasian patients. Particularly for Asian patients with Child-Pugh C, the nonlinear PK of PAA could result in an over-proportional increase in plasma PAA concentrations during 5 days of treatment that could exceed the margin that was confirmed to be safe in study HE209.

Phenylacetylglutamine is excreted in urine, therefore renal dysfunction is expected to alter PAGN plasma exposure. Our observations agreed with the report of Stravitz et al. [11]. However, elevated PAGN plasma concentration due to renal dysfunction did not alter PAA exposure and ammonia level reduction in plasma circulation (Fig. 2), confirming that the conversion of PAA to PAGN is irreversible. Therefore, an elevated PAGN plasma concentration will not alter the rate of conjugation of PAA to glutamine.

Mokhtarani et al. [13] investigated the correlation between PAA concentrations and neurotoxicity by evaluating data from a crossover study in 98 healthy adults, a 4-week safety and dose-escalation study in patients with cirrhosis, and a 16-week, randomized, placebo-controlled multi-center study in 178 patients with cirrhosis and HE. The relationship between PAA concentrations and neurologic AEs appeared to exist in healthy volunteers. However, the odds of experiencing a neurologic AE did not increase with elevated PAA concentrations in patients with urea cycle disorders or HE. The author suggested that the variation in relationships between PAA exposure and CNS AEs could have been attributed to intrinsic differences among populations. Therefore, PAA threshold values from other populations (e.g., the $500-\mu \mathrm{g} / \mathrm{mL}$ threshold for oncology patients) $[5,6]$ may differ and should not be extrapolated to patients with chronic liver disease (cirrhosis) and HE. Our current analysis showed no correlation between PAA exposure and CNS AEs in both healthy individuals and patients with liver cirrhosis/HE

In summary, the present study confirmed the findings that PAA exposure was not correlated with neurologic AEs in patients with HE. Body weight/body surface area and liver function significantly impacts the PK of PAA. When a flat dose of L-OPA across all body weight is chosen, attention should be paid to those patients with a low body weight and severe hepatic impairment. Although renal impairment significantly increases PAGN plasma exposure, it has no impact on AEs or on ammonia levels.

Supplementary Information The online version contains supplementary material available at https://doi.org/10.1007/s40262-021-01047-5.

Acknowledgments Editorial support for the preparation of this manuscript was provided by Peloton Advantage, LLC, an OPEN Health company, Parsippany, NJ, USA, and funded by Mallinckrodt Pharmaceuticals.

\section{Declarations}

Funding This study was sponsored by Mallinckrodt Pharmaceuticals, which participated in the review of and decision to publish this manuscript.

Conflicts of Interest/Competing Interests Xiaofeng Wang, Carmen Mak, and Nagaraju Poola are former employees of Mallinckrodt Pharmaceuticals. Jack Tseng and Regis A. Vilchez are current employees of Mallinckrodt Pharmaceuticals. The study sponsor was involved in the study design and data collection, analysis, and interpretation.

Ethics Approval An institutional review board approved each study protocol.

Consent to Participate All participants provided written informed consent prior to initiation of study-related procedures.

Consent for Publication Not applicable (no personal data were reported).

Availability of Data and Material Discussion of statistical endpoints and analysis are included in the article. Summary aggregate (basic) results (including adverse event information) and the protocol for study HE209 will be available on clinicaltrials.gov (NCT01966419) when required by regulation. Individual de-identified patient data will not be disclosed. Requests for additional information should be directed to the company at medinfo@mnk.com. 
Code Availability Phoenix ${ }^{\circledR}$ WinNonlin ${ }^{\circledR}$ Version 6.4 (Certara Inc., Princeton, NJ, USA), NONMEM Version 7.4 (ICON, Ellicott City, MD, USA), and R Version 3.5 .

Authors' Contributions XW conducted the pharmacokinetic and exposure-adverse event analyses, interpreted the outcome of the analyses included in the manuscript, and was a key contributor to the design of the two clinical studies. JT participated in the acquisition/collection of the data and interpretation of the data. CM reviewed and interpreted the data. NP participated in the data summary, review, and interpretation. RAV designed and analyzed the study. All authors drafted the manuscript or revised it critically for important intellectual content and approved the version to be submitted.

Open Access This article is licensed under a Creative Commons Attribution-NonCommercial 4.0 International License, which permits any non-commercial use, sharing, adaptation, distribution and reproduction in any medium or format, as long as you give appropriate credit to the original author(s) and the source, provide a link to the Creative Commons licence, and indicate if changes were made. The images or other third party material in this article are included in the article's Creative Commons licence, unless indicated otherwise in a credit line to the material. If material is not included in the article's Creative Commons licence and your intended use is not permitted by statutory regulation or exceeds the permitted use, you will need to obtain permission directly from the copyright holder. To view a copy of this licence, visit http://creativecommons.org/licenses/by-nc/4.0/.

\section{References}

1. Vilstrup H, Amodio P, Bajaj J, et al. Hepatic encephalopathy in chronic liver disease: 2014 Practice Guideline by the American Association for the Study of Liver Diseases and the European Association for the Study of the Liver. Hepatology. 2014;60:715-35.

2. Olde Damink SW, Jalan R, Redhead DN, Hayes PC, Deutz NE, Soeters PB. Interorgan ammonia and amino acid metabolism in metabolically stable patients with cirrhosis and a TIPSS. Hepatology. 2002;36:1163-71.

3. van Straten G, de Sain-van der Velden MGM, van Geijlswijk IM, et al. Saline is as effective as nitrogen scavengers for treatment of hyperammonemia. Sci Rep. 2017;7:13112.
4. Buphenyl [package insert]. Scottsdale (AZ): Ucyclyd Pharma, Inc.; 2008.

5. Ravicti [package insert]. Lake Forest (IL): Horizon Pharma USA, Inc.; 2019.

6. Ravicti [summary of product characteristics]. Stockholm: Immedica Pharma; 2020.

7. Meijer AJ, Lamers WH, Chamuleau RA. Nitrogen metabolism and ornithine cycle function. Physiol Rev. 1990;70:701-48.

8. Clinical laboratory reference values. In: Laposata M, editor. Laboratory medicine: the diagnosis of disease in the clinical laboratory. 2nd ed. New York (NY): McGraw-Hill Education; 2014: p. xvii-xxxi.

9. Jalan R, Wright G, Davies NA, Hodges SJ. L-Ornithine phenylacetate (L-OPA): a novel treatment for hyperammonemia and hepatic encephalopathy. Med Hypotheses. 2007;69:1064-9.

10. Misel ML, Gish RG, Patton H, Mendler M. Sodium benzoate for treatment of hepatic encephalopathy. Gastroenterol Hepatol (N Y). 2013;9:219-27.

11. Stravitz RT, Gottfried M, Durkalski V, et al. Safety, tolerability, and pharmacokinetics of 1-ornithine phenylacetate in patients with acute liver injury/failure and hyperammonemia. Hepatology. 2018;67:1003-13.

12. Thibault A, Samid D, Cooper MR, et al. Phase I study of phenylacetate administered twice daily to patients with cancer. Cancer. 1995:75:2932-8.

13. Mokhtarani M, Diaz GA, Rhead W, et al. Elevated phenylacetic acid levels do not correlate with adverse events in patients with urea cycle disorders or hepatic encephalopathy and can be predicted based on the plasma PAA to PAGN ratio. Mol Genet Metab. 2013;110:446-53.

14. Rahimi RS, Safadi R, Thabut D, et al. Efficacy and safety of ornithine phenylacetate for treating overt hepatic encephalopathy in a randomized trial. Clin Gastroenterol Hepatol. 2020. https://doi. org/10.1016/j.cgh.2020.10.019.

15. Buphenyl [Japanese package insert]. Tokyo: Orphan Pacific DI Center Co., Ltd.; 2015.

16. Monteleone JP, Mokhtarani M, Diaz GA, et al. Population pharmacokinetic modeling and dosing simulations of nitrogen-scavenging compounds: disposition of glycerol phenylbutyrate and sodium phenylbutyrate in adult and pediatric patients with urea cycle disorders. J Clin Pharmacol. 2013;53:699-710. 